\title{
VORWORT DER HERAUSGEBER ZUR DEUTSCHEN AUSGABE
}

Im Vorwort zur dritten russischen Auflage des fünften Bandes dieser zehnbändigen Gesamtdarstellung der modernen theoretischen Physik wird zur Neugliederung der Statistischen Physik in Teil 1 (Band V) und Teil 2 (Band IX) folgendes gesagt: „Im neunten Band wird eine detaillierte Darstellung der Methode der Grersschen Funktionen und der Diagramm-Technik gegeben, die die Entwicklung der statistischen Physik während der letzten zwanzig Jahre wesentlich bestimmten. Die Abtrennung dieser (und auch einer Reihe anderer) Fragen wird nicht nur dadurch diktiert, daß ein Einbeziehen in den fünften Band zu einer übermäßigen Ausdehnung seines Umfanges und zu einer wesentlichen Anderung seines ganzen Charakters führen wüirde. Es handelt sich auch darum, daß diese Fragen ihrer Natur nach auch in einem starken Maße mit der Hydrodynamik und der makroskopischen Elektrodynamik zusammenhängen (es ist z. B. sinnvoll, sich bei der Behandlung der mikroskopischen Theorie der Supraleitfähigkeit auf die schon bekannte makroskopische Theorie dieser Erscheinung zu stützen). Aus diesem Grunde soll das neue Buch seinen Platz in der Reihenfolge der Bände dieses Lehrbuches nach der Mechanik und Elektrodynamik der Kontinua finden.“

Der gesamten Darstellung in diesem Band liegt die LANDAJ-Theorie der Quantenflüssigkeiten als Ausgangspunkt und Rahmen zugrunde; solche speziellen Modelle wie wechselwirkungsfreie Quantengase werden lediglich zur Illustration der allgemeinen Theorie herangezogen. Immer wieder beeindruckt, wie es den Autoren - so auch in diesem Band - gelingt, den Bogen von der allgemeinen Theorie über die Behandlung bekannter Beispiele bis zu modernen Anwendungen zu spannen und gegenüber dem Mathematisch-Formalen das Physikalisch-Anschauliche deutlich hervortreten zu lassen. Die Vielfalt der behandelten Probleme aus der Quantentheorie der kondensierten Materie sowie Gründlichkeit und Originalität ihrer Darstellung machen dieses Werk so wertvoll.

Herrn Prof. E. M. LIFschrTz gebührt unser herzlichster Dank für seine freundliche Unterstützung bei der Vorbereitung der deutschen Ausgabe. Die Ubersetzung wurde in bewährter Weise von Herrn Dr. E. JäGER besorgt. Beim Korrekturlesen leistete Herr Dr. M. Hrasschold wertvolle Hilfe.

Dresden, im September 1979

P. Zrasohe, H. Eschria 